\title{
Applications of Artificial Intelligence in Transport: An Overview
}

\author{
Rusul Abduljabbar *, Hussein Dia *, Sohani Liyanage and Saeed Asadi Bagloee \\ Department of Civil and Construction Engineering; Swinburne University of Technology, \\ Hawthorn, VIC 3122, Australia; sliyanage@swin.edu.au (S.L.); sasadibagloee@swin.edu.au (S.A.B.) \\ * Correspondence: rabduljabbar@swin.edu.au (R.A.); hdia@swin.edu.au (H.D.); \\ Tel.: +61-432-299-979 (R.A.); +61-392-145-280 (H.D.)
}

Received: 4 November 2018; Accepted: 24 December 2018; Published: 2 January 2019

\begin{abstract}
The rapid pace of developments in Artificial Intelligence (AI) is providing unprecedented opportunities to enhance the performance of different industries and businesses, including the transport sector. The innovations introduced by AI include highly advanced computational methods that mimic the way the human brain works. The application of AI in the transport field is aimed at overcoming the challenges of an increasing travel demand, $\mathrm{CO}_{2}$ emissions, safety concerns, and environmental degradation. In light of the availability of a huge amount of quantitative and qualitative data and $\mathrm{AI}$ in this digital age, addressing these concerns in a more efficient and effective fashion has become more plausible. Examples of AI methods that are finding their way to the transport field include Artificial Neural Networks (ANN), Genetic algorithms (GA), Simulated Annealing (SA), Artificial Immune system (AIS), Ant Colony Optimiser (ACO) and Bee Colony Optimization (BCO) and Fuzzy Logic Model (FLM) The successful application of AI requires a good understanding of the relationships between $\mathrm{AI}$ and data on one hand, and transportation system characteristics and variables on the other hand. Moreover, it is promising for transport authorities to determine the way to use these technologies to create a rapid improvement in relieving congestion, making travel time more reliable to their customers and improve the economics and productivity of their vital assets. This paper provides an overview of the AI techniques applied worldwide to address transportation problems mainly in traffic management, traffic safety, public transportation, and urban mobility. The overview concludes by addressing the challenges and limitations of AI applications in transport.
\end{abstract}

Keywords: Artificial Intelligence; Genetic algorithms; Simulated Annealing; Artificial Immune system; Ant Colony Optimiser; Bee Colony Optimization; public transport; Auto Urban Mobility; traffic management

\section{Introduction}

Artificial intelligence (AI) is a broad area of computer science that makes machines function like a human brain. It is used to address issues that are difficult to clarify using traditional computational techniques. AI was first discovered in 1956 by John McCarthy but failed to achieve its objectives [1], and the lack of technology innovations made it less promising. From 1960 to 1970, researchers explored AI through the Knowledge-based system (KBS) and Artificial Neural network systems (ANNs) [1]. The KBS systems are computers that provide advice using pre-determined rules, according to the knowledge presented to it by humans. The ANNs, on the other hand, are systems of neuron connections designed in various layers, modelled after the human brain which have been used in medicine, biology, and language translation engineering, law, manufacturing, etc. [2,3]. During that period of time, interest in AI diminished due to limited applications of ANNs and lack of data until the 1980a [4]. 
Since the 1980s, many research was conducted to minimize the error of prediction through a method dubbed as gradient descent. This method is referred to as a Backpropagation algorithm for the training of the ANNs and it was applied to solve problems in different domains using few hidden layers $[5,6]$. Today, the availability of data has introduced the concept of machine learning as a subcategory to AI. Machine learning implies coding the computers to behave like a human brain instead of teaching them everything. It provides the computers with access to big data and extract important features from them to solve complicated problems $[7,8]$.

ANN is the most distinguished AI method used in different applications. One of the first and most common types of ANNs is the Feedforward Neural Network in which the data moves in one direction from the input layer to the hidden layer to the output layer. Other types of ANNs are Convolutional Neural Network (CNN) [9-11] and Recurrent Neural Network [12-14]. The CNN performs better for image processing tasks while RNN Processes a sequence for the input data to become well-suited for many application such as; language, writing and text recognition. They are often referred to as Deep Learning Techniques due to the multiple hidden layers structured in their architectures.

There are many uncertainties and gaps within the data that cannot be solved using traditional techniques. Therefore, AI uses those uncertainties and model a relationship between the cause and effect of different real-life scenarios by combining the available data with assumptions and probabilities for a better analysis [15].

Transportation problems become a challenge when the system and users' behaviour is too difficult to model and predict the travel patterns. Therefore, $\mathrm{AI}$ is deemed to be a good fit for transportation systems to overcome the challenges of an increasing travel demand, $\mathrm{CO}_{2}$ emissions, safety concerns, and environmental degradation. These challenges arise from the steady growth of rural and urban traffic due to the increasing number of population, especially in the developing countries. In Australia, the cost of congestion is expected to reach 53.3 billion as the population increase to 30 million by 2031 [16]. In Melbourne, Australia alone, more than $640 \mathrm{~km}$ of arterial roads are congested during peak time with a $\mathrm{CO}_{2}$ emission of 2.9 tons per year [17]. Many researchers in the 21st-century attempt to accomplish a more reliable transport system with less effect on people and the environment using cost-effective and more reliable by AI techniques. It has a potential application for the road infrastructure, drivers, road users, and vehicles.

The AI applications in transport have been developing and implementing in a variety of ways. Among those, this research paper aims to address three main examples. (i) The use of AI in corporate decision making, planning, and managing. This is important to overcome the issue of a continuously rising demand with limited road supply. This includes better utilization of accurate prediction and detection models aiming to better forecast traffic volume, traffic conditions, and incidents. (ii) Applications of AI aiming to improve public transport is also discussed. It is due to the fact that public transportation is regarded as a sustainable mode of mobility. (iii) The next promising AI application in transport is connected and autonomous vehicles, which aims to enhance productivity by reducing the number of accidents on highways. The self-driven cars and small-scale autonomous bus trials that have been initiated, most prominently in Finland, Singapore, and China are overviewed in this paper.

The remainder of this paper is divided as follows: Section 2 discusses the application of AI in transport. This section is subdivided into the application of AI for planning, designing and decision making, public transportation, intelligent self-driving cars. It also illustrates real-time incident detection and future traffic state prediction. Section 3 shows that the future of AI is focused on Deep Learning. Section 4 explains the future research work by the authors while Section 5 provides a conclusion of this paper review.

\section{Applications of AI in Transport}

In many cases, it is hard to fully understand the relationships between the characteristics of the transportation system; therefore, AI methods can be presented as a smart solution for such complex 
systems that can't be managed using traditional methods. Many researchers have demonstrated the advantages of $\mathrm{AI}$ in transport. An example of that includes transforming the traffic sensors on the road into a smart agent that detects accidents automatically and predicts future traffic conditions [18]. Also, there are many AI methods used in transport such as ANNs. ANNs can be used for road planning [19], public transport [20,21] Traffic Incident Detection [22-25]; and predicting traffic conditions [26-33]. It is classified into supervised and unsupervised learning methods. Supervised methods include Support Vector Machine (SVM), Probabilistic Neural Network (PNN), Radial Basis Network (RBN), K-Nearest Neighbors and Decision Tree, etc. while unsupervised NNs include greedy layer-wise and cluster analysis.

Many transportation problems lead to an optimization problem that needs bespoke algorithms to make computational analytics easy to solve. They are highly advanced computational algorithms referred to as raster algorithms. The Genetic Algorithm (GA) is an example of those algorithms. It is based on the evolutionary biological concept. It solves complex optimizations problems based on "survival of the fitness" concept and it is a good tool to use in the urban design networks [34-37]. Another algorithm is Simulated Annealing (SA) which is obtained from by simulating the process of annealing of metal [34,38-40]. Ant Colony Optimiser (ACO) is also an AI algorithm developed based on the behaviour of a group of real ants following their path from the nest to food source [41,42]. An artificial Immune system (AIS) which is modelled based on the human immune system $[34,43,44]$. Bee Colony Optimization (BCO) which solves a hybrid complex optimization problem [44-47]. ACO and BCO are part of swarm intelligence systems [41-44]. Swarm intelligence is an AI system which is inspired by ants and bees working together as a group to reach to an optimised solution. The intelligent computational analytics of these system are able to represent uncertainty, imprecision and vague concepts, hence these techniques are used for route optimization problems in transport [48-51]. Another optimisation technique is Fuzzy Logic Model (FLM). It is applied to solve shortest path optimization [52]. The performance of FLM is compared with Logistic Regression Model (LRM) by [53] when developing a route choice model, and FLM outperformed. Therefore, intelligent techniques such as FLM, GA, ANN, ACO are suitable for prediction, reasoning, and adaptability. Therefore, these are used to solve optimization problems which involve dynamic traffic situations and events. Another novel software paradigm has introduced based on AI theories, called Agent-Based Software Engineering (ABSE). ABSE is capable of allowing the dynamic approach to identifying shortest path through the formation of multi-criteria and multi scenarios [54].

Also, NNs were utilised to integrate the system with the aforementioned algorithms for better results $[22,49,55]$. For example, references [50,51] showed another tool used for AI as a software and hardware implementation for automated vehicles and trip planning. It is important for transport authorities to determine when and how to use these technologies to make a rapid improvement in relieving congestion, making travel time more reliable to their customers and improve the economics and productivity of its vital assets.

\subsection{AI in Planning, Designing and Controlling Transportation Network Structures}

The objective of planning is to identify the community needs and decide on the best approach to meet this demand while utilizing the impact of social, environmental and economic in transportation. Designing an optimal road method for transport planning is part of the Network Design Problem (NDP) [56]. It can be a Continuous problem when the capacity of existing infrastructure changes (extend lane width, median and shoulder area), a Discrete problem is identified when adding more infrastructure and a Mixed of Continuous and Discrete problems. Previous researches in the 90s focused on NNs for road planning, designing and modelling. For example, reference [57] modelled the spatial relationship between transportation and land-use planning by using a parallel neural network system. After that, research was more focused on raster algorithms which are more preferred for urban planning because they don't require existing links and nodes to find the optimal path [58]. Today, emerging huge amount of data with advanced algorithms become the interest of most research by 
using Machine-learning to create patterns among the data. Reference [34] addressed the continuous NDP problem as a Bi non-linear model assignment with two levels. They used GA and SA algorithms and compared their efficiency on a simulated network. When the demand is low, SA finds optimal value with less computational effort than GA. However, when more computations are done by GA, it can reach a better optimal solution. This result opposed to [37], in which the authors argued that GA provides better results than SA with less computational time. However, the model considered in this paper was a single-level linear model for a Continuous NDP problem.

Another study [21] modelled the safety Management plan for Ankara city in Turkey using ANN and Genetic algorithms. The results obtained from ANN model were better than GA with less error involved. While [59] used the ant colony algorithm for the design of an optimal vehicle path. Moreover, reference [58] combined Cellular Automata (CA) which is a spatial simulation method with Ant Colony optimization technique (ACO). The results showed a planning improvement for urban development based on the simulated industrial land use patterns for several years in China. Furthermore, reference [19] focused on finding a good transportation system management and safety plan to balance the transport demand with a proper distribution of highways, railways, and airways. The author used ANN for accident and injury prediction for a highly congested route from Istanbul to Ankara. Last, reference [33] compared between GA, SA and AIS algorithms to find the best modification for an existing network structure considering it as a Mix NDP problem in Poland. The results demonstrated that SA performs worse than GA and AIS for this class of problem.

In addition, planning of routes for vehicles is important to avoid congestion and delays in travel times. Many Authors concluded that ant colony algorithm is a promising solution for the vehicle routing problem [57,60-62]. While [63] focused on solving a routing and wavelength problem using $\mathrm{BCO}$ Algorithm. The problem means choosing a path within a network and assigning a wavelength to that path for each connected node to maximize the number of connections established between the nodes in the network. Moreover, recent research focused on utilizing microscopic traffic data for modelling and identifying security breaches and for traffic control systems and management plans of roadways [64]. In terms of finding best paths for public transport users, reference [65] suggests to learn and update the real time path generation system according to the preferences of travellers. Also, to use a utility-based approach which concentrates on different attributes of paths and parameters per each public transport user.

An area where AI applications have also seen rapid developments is Intelligent Transport Systems (ITS). These systems aim to alleviate congestion and improve driving experience using a variety of technologies and communication systems. They capture important data that can be integrated with machine learning technology. For example, deep reinforcement learning has been used for real time optimisation of traffic control policies embedded in large scale ITS systems [66]. Similarly, a deep learning system to empower ITS devices with functions for signal processing and fast computing analytics has also been proposed [67]. In the future, data complexity will increase as ITS continues to develop, hence, deep learning techniques will be essential to find patterns and features in these data to achieve a more connected transportation systems. In another example [68], genetic algorithm and fuzzy methods were used to control the traffic signal systems automatically at intersections. The ITS system proposed, 'NeverStop', utilised RFID sensors and the findings showed that this system was effective to reduce average waiting times for vehicles. Also, two NNs systems [69] were developed to manage the road more efficiently based on microscopic simulated data. The first system is to control the traffic signal and the second one is to predict future traffic congestion. While [70] demonstrated the feasibility of using NNs to control the traffic by proposing a multi-layer NNs system evaluated in three intersections networks [70].

ANNs are also effective to use in signal traffic control. Reference [71] Developed two NNs systems to manage the road more efficiently based on microscopic simulated data. The first system is to control traffic signal and the second one is to predict future traffic congestion. While, reference [72] 
demonstrated the feasibility of using NNs to control the traffic by proposing a multi-layer NNs system evaluated in three intersections networks [72].

Moreover, reinforcement learning NNs is used to update the parameters and cycle length of the system as the flow changes periodically $[64,65]$. AI is a dynamic research area that keeps on improving and new methods and applications are introduced frequently to utilize the strength of AI to improve the planning, decision making and management of the road.

\subsubsection{Incident Detection}

Many attempts were established to identify, the time, location and the severity of an incident to support traffic managers to mitigate congestion. These attempts are ranging from manual reports to automated algorithms to Neural Networks. Manual reports that are written by humans can have a delay in detecting incidents and cost-effective. On the contrary, algorithms can measure the flow characteristics before and after the incident through data collected from sensors along the road. Algorithms for incident detection has been first implemented using statistical techniques such as California Algorithm. However, it is difficult to use an algorithm on arterial roads, because of the street parking and traffic signals. For this reason, algorithms have been developed to neural networks approaches. A classification neural network algorithm was evaluated to detect incident occurrence in a freeway [22]. Other research considered finding a good software package to detect all objects of vehicles on real-time [73]. The author suggested using AdaBoost software for accurate image detection. Reference [24] proposed IMM ENKF algorithm to detect incidents for hybrid state problems. Then, a more accurate algorithm named "Efficient Multiple Model Particle Filter (EMMPF) was developed to detect incidents on the highway using both simulated and field data [23]. Moreover, incidents in real time can be detected from social media as discussed in [74]. It showed that Twitter is a cost-effective and efficient technique to acknowledge incident occurrence on both, freeway and arterial roads.

\subsubsection{Predictive Models}

The rapid development of intelligent transport systems (ITS) has increased the need to propose advanced methods to Predict traffic information. These methods play an important role in the success of ITS subsystems such as advanced traveller information systems, advanced traffic management systems, advanced public transportation systems, and commercial vehicle operations. Intelligent predictive systems are developed using historical data extracted from sensors attached to the roads. Then, these data becomes an input to machine learning and AI algorithms for a real-time, short-term and long-term predictions [75].

In the past, research focused for short-term flow prediction by using simple feedforward neural network. Reference [28] integrated a neural network system with one hidden layer to the overall urban traffic control system. The author demonstrated the strong possibility of predicting traffic flow up to $1 \mathrm{~min}$ by using simulated data only. While, reference [25] used field data from a $1.5 \mathrm{~km}$ section of a highway in Queensland, Australia. An object-oriented neural network model was developed with a time-lag recurrent network (TLRN). The model was able to predict speed for 5 min into the future with $90-94 \%$ accuracy. Also, when using speed and flow as an input to the network, it predicted travel time up to $15 \mathrm{~min}$ with that same accuracy as speed prediction.

In another example [29], the authors developed a deep neural network to predict the traffic flow up to $60 \mathrm{~min}$ into the future. The traffic flow data were collected from freeways across California. The authors used an unsupervised stack of auto-encoders named (SAE) model and train it by using a greedy layer-wise algorithm. It extracts important features of traffic flow as each output is fed back as an input to the network. Then, a supervised logistic regression layer is applied for prediction.

The model showed a superior performance for nonlinear spatial and temporal traffic data correlation when compared to other machine learning methods such as SVM and Multi-layer NN/Back Propagation NN. While [26] proposed unsupervised Deep Belief Network (DBN) trained using a greedy layer-wise algorithm to learn important features from the flow pattern. Then applied a regression 
supervised layer for prediction. However, the authors suggest a multitasking regression layer with a weight grouping method to join multiple tasks together and train the model. This showed an improvement in the accuracy of the model. In addition, reference [31] developed a deep learning model by using Field data collected from California to capture the long-term traffic flow prediction. The model was successful in extracting spatial features of traffic flow by using the convolutional neural network and temporal traffic flow features from Recurrent Neural Network.

Another AI technique to predict the traffic flow is presented by [30]. The authors developed a simple recurrent neural network for short-term forecasting using Jordan's neural network. It differs from conventional feedforward network by the addition of a context layer. This context layer stores the previous information and acts as a memory box. Then the stored information at $(\mathrm{t}-1)$ is feed-forwarded to the hidden layer along with the input at a time (t). This helps the network to predict the subsequence; for this reason, it is sometimes called "Jordan's Sequential Network". The data input was traffic volume taken from Ireland road traffic control. And the output is the future traffic flow. This network is trained as a feed-forward neural network with back propagation algorithm to mitigate the error. However, the authors proved that the network provides better accuracy when the number of neurons in the hidden layer is double the number the input neurons. Also, with a learning rate of 0.5 and decreasing number of iteration gives a prediction of flow accuracy between $92-98 \%$. Since this model is considered as a first order system which provides inaccurate prediction when computing higher order dynamics, reference [76] suggested to frequently linearize the network at each operating point online to make algorithm computation simpler. Another type of recurrent network is Elman Network [77]. In addition, a Pattern-Based Strategy (PBS) was developed by ATOS Scientific Community. Pattern recognition can be divided into, first, Supervised learning in which labelled data used with a correct output. Second, Unsupervised learning where unlabelled data are used to find a pattern and find the correct output; and Semi-supervised learning with little-supervised data used with a huge amount of unlabelled data for pattern recognition analysis [78]. Data were collected from the city of Berlin for speed and flow rate and the results demonstrated a forecasting accuracy of $93 \%$ for speed and $86 \%$ for the flow rate.

In order to build a robust predictive model, it is important to acknowledge three phases as seen in Figure 1.

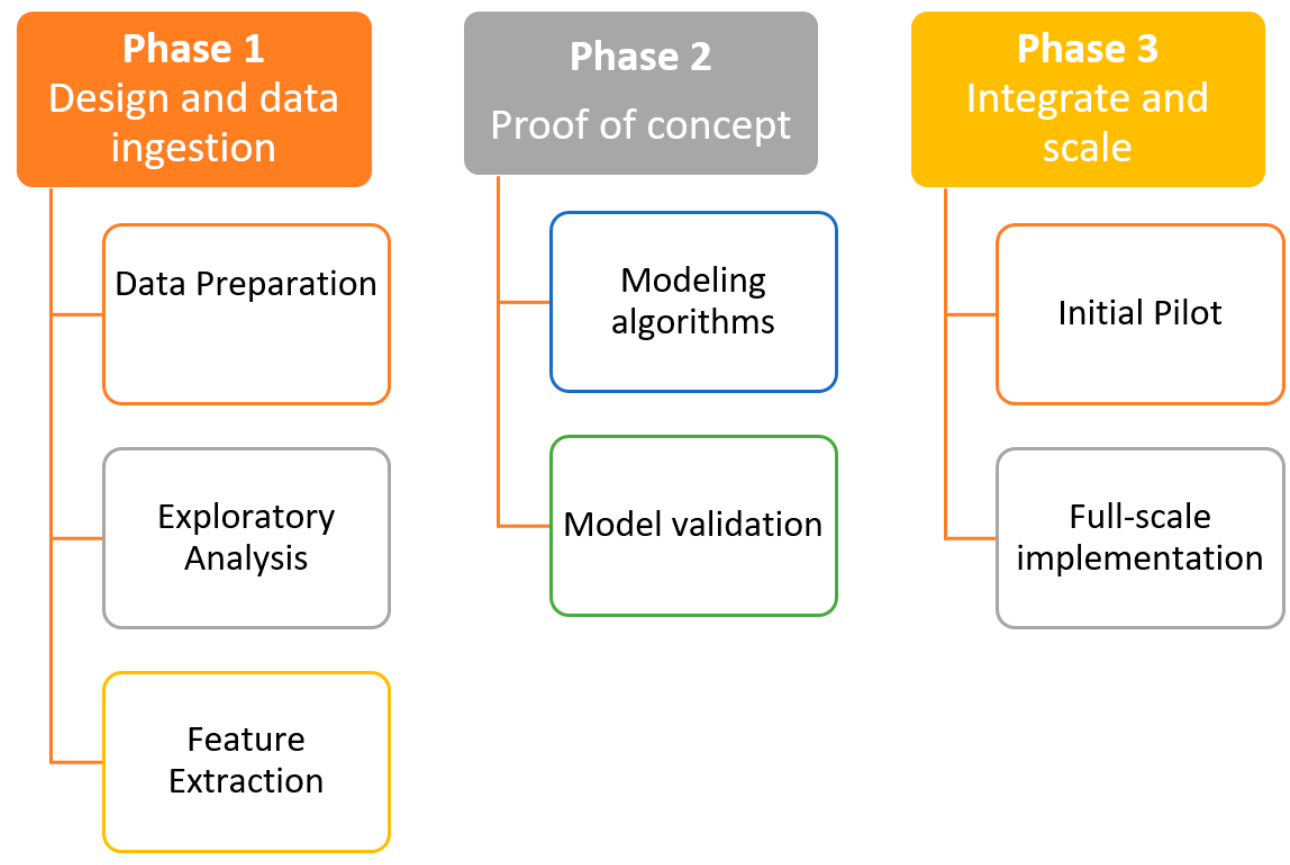

Figure 1. Three-Phases Approach to develop advanced predictive model—adapted from [79]. 
In phase 1, all data sources must be evaluated and used for incorporating the advanced model into the industry. This phase is important for the initial assessment of the asset performance. Relatively, in the proof of concept phase, many models can be chosen for a more critical assessment of the performance by identifying failures modes and the time required for the overall life-cycle of the project. Moreover, the last phase of the diagram represents the real-time prediction information on asset performance as the model in this phase should be constantly updated and scaled to give the best results.

In [80] the authors use demographic and Geographic data to predict future mobility demand in Switzerland. This demand estimation is important to make a decision on planning and future techniques that are most needed to manage a more effective transportation system. They, first cluster the population data based on their daily route choices. Then, a decision tree is used to classify these data and support vector machine to improve to extract more important features from the data. They are both supervised NNs. A decision tree uses large numbers of de-correlated decision trees to create a forest. Higher accuracy is achieved by the increasing number of trees. It works by classifying each tree $(x)$ based on its attributes. The tree votes for $(x)$ and the classification with most votes is chosen by the forest. While SVM classifies the inputs based on maximizing the margins between the data. Then, they use machine learning algorithms with respect to daily distance travelled by a vehicle for the estimation of the future demand. In another study [65], the authors investigate the methods applicable for real time short term prediction of public transport users waiting at bus stops and also on-board passengers. This information will help operators to control transit trips more effectively. It also helps travellers to decide on the best route during congested hours. The communication of information requires integration between public transport systems and ITS which in turn will enhance the forecasting tools for advanced traveller information systems and operation controls.

Similarly, recent ridesharing services such as Uber and Didi Chuxing have increased the possibility of collecting massive amounts of data. AI can benefit from these data to predict passenger demand effectively to avoid empty vehicles which in return will reduce congestion and energy consumption [81]. A deep learning model that considers Multi-View, Spatial and Temporal (DMVST) Network is proposed by [81]. The authors collected large scale ridesharing demand requested data from DiDi Chuxing in the city of Guangzhou in China. They combined Local CNN that captures local regions in relations to their surrounding area and Long Short Term Memory network (LSTM) to model temporal features. The results demonstrated a superior performance of this recommended model. Similarly, reference [82] predicted taxi demand for taxi services in Tokyo, Japan by using Multi-layer perceptron Neural Network. They collect data from Taxi Probe system in which the taxis are equipped with sensors that record information (e.g., Location of the taxi). The results showed that the historical demand data of $4 \mathrm{~h}$ with 50 neurons in the hidden layers provided better prediction accuracy. Similarly, another study [83] determined the performance of taxis by selecting the most important feature from a taxi pattern using L1-Norm SVM. Also, another study [84] forecasted taxi travel demand by using deep learning techniques using taxi datasets from New York City, USA. The DNN outperformed other machine learning methods, however, the right architecture must be identified to get accurate results.

Finally, AI can play an important role to prevent urban road accidents and reduce the impacts of accidents. The reasons behind vehicle accidents varies in space and time. Hence, AI can capture the spatial-temporal pattern of accidents in databases and identify patterns for which mitigation strategies can be designed. For example, a study [85] used deep recurrent neural network approach to predict the risk of traffic accidents by analysing the spatial and temporal patterns from a traffic accident database in Beijing, China. The results showed that this method was effective and can be applied to warn people around hazardous locations. Similarly, another study [86] developed a Stack Denoise Autoencoder Simulation model to predict the risk level of traffic accidents. In another study [87], the authors discovered that machine learning techniques (k-Clusters and priori algorithm) can identify valuable hidden patterns from a vehicle crash dataset of historical accidents. For example, according to [88], the most important factors associated to the fatal severity of an accident in United Arab Emirates 
were: gender (male with highest level of accidents), age (between 18-30 years old mostly involved in accidents), and collision type (car to pedestrian collision is the common one in accidents) and location of an accident (right angles). These factors were determined using machine learning algorithms such as: Decision Tree and MLP.

\subsection{Application of AI in Aviation and Public Transportation}

The development of computer science has made field data collection more reliable and approachable. Public Transport data are dense and unstructured; therefore, it needs to be modelled properly to take full advantage of these data.

\subsubsection{Aviation}

AI has been acknowledged to manage the flight journey more effectively. AI can help in Technology (Machine Learning), software/hardware and Application (Intelligent Maintenance, Flight Route Optimization). In [89] a system called (PLADS) was developed to extract information from highly dense aviation reports and modify it to support vector machine and SA algorithm systems. It showed that SVM gives good results for this type of classification. Whereas, reference [20] showed that the unsupervised machine learning algorithm is reliable to use to increase safety when an airplane is landing. Relatively, reference [55] assessed the safety of the plane by checking the engine on-board using the Probabilistic neural network (PNN). The training of PNN for the network is similar to MLP but with using a radial basis function instead of the linear function and more neurons are needed in the hidden layer of RBN. Moreover, reference [90] developed an automated supervised Random Forest system to detect aviation turbulence more accurately. This system can help the pilot to avoid deviating from the pre-determined route, minimize fuel cost, and enhance air control management. Last, reference [91] showed that using ANN is important to enhance the monitoring system of the gas turbine during flights. The author suggests that each element in the system should be assigned to a diagnosing NN to reach the highest accuracy needed to enhance the monitoring system. PNN, MLP, SVM, and RBN are compared for engine 3 which is for a transport aircraft for single and multiple neural network systems. The results were as follows: Multiple RBN has the highest probability computed of 0.7787 in mode 1 and 0.7643 in mode 2, while single PNN has the lowest of 0.7287 for mode 1 and 0.7456 for mode 2 . However, equal performances were acknowledged from the NNs and SVM in two modes.

\subsubsection{Shared Mobility}

In dense urban cities, especially, sharing economy in mobility has become promising as this lead to a reduction in inner-city traffic congestion and pollution, with its ability to provide strategies to remove the single or less occupant personal vehicles on the road. Therefore, this enables a win-win strategy, which provides short-term socio-economic benefits, in addition to efficient long-term sustainable solutions through new adoptions to mitigate traffic issues and environmental impacts.

In general, sharing economy is defined by [92] as,

"ICT-enabled platforms for exchanges of goods and services drawing on non-market logics such as sharing, lending, gifting and swapping as well as market logic; renting and selling"

The momentum of the sharing economy mainly lies in Transportation and Accommodation sectors and the pioneers of the sharing economy market are Uber and Airbnb [93]. In the transportation sector, the emerging of sharing economy has a potential interest towards developing business models to emerge new-shared mobility services to provide a sustainable transportation system and address the gap of fitting the demand to the supply more effectively [94]. Specifically, sharing bikes, cars, and rides on an on-demand basis has driven the attention of the majority of users. These shared mobility options also address the major shortcoming of "first-mile, last-mile." 
The shared mobility models such as bike sharing and car sharing have existed for decades, figuratively, the first bike-sharing models have emerged the Amsterdam in 1960s [95]. However, with the advancements in information and communication technologies, the recent advances and developments of disruptive innovations in shared mobility have become possible at a scale. In addition, these app-based platforms coordinate the on-demand vehicles and pairing to share rides for a long-term efficient transport system.

Shared mobility is benefited by the AI technology to improve the customer experience and streamline their businesses. The providing of personalized customer experience to the users has become possible with the integration of AI and shared mobility. For example, the Uber has provided personalized rider experience by suggesting rider destinations based on the user's ride history. In addition, the Uber has introduced route-based pricing, which uses AI to predict how much riders are willing to pay based on the parameters such as destination, time of day and location. Moreover, the Uber operators are using AI to identify the fraudulent drivers and prevent fraudulent activities. The future of shared mobility is pictured with driverless cars, which uses AI and sensor technologies. The autonomous fleets are more profitable and this could supplant 6.2 millions of drivers globally, by the year 2030 [96]. These advancements of AI in transport also made shared transport operators like Uber, to plan the route efficiently, estimate travel time including pick up and drop off times accurately. This leads to enhance the reliability and efficiency of shared public transportation. Furthermore, passenger demand prediction for public transportation based on past ride data and environmental information has become possible with access to big data and ANN. An algorithm, i.e., a location recommendation system that targets the potential passengers during the reservation when buses are idle is introduced by [97]. With the use of machine learning knowledge, Q-learning [98] has found an effective pick-up point selection process.

\subsubsection{Buses}

Since Buses play an important role in the public transportation sector, many researchers have been conducted to make bus journeys and destinations more safe and reliable. Whereas $[99,100]$ use Hybrid Ant Colony Algorithm to manage bus schedule. They both are efficient and reliable for the optimum scheduling of bus drivers. Also, ANNs are efficient to use to reduce passenger waiting time by predicting bus arrival times [101,102]. Application in buses has also been extended to the design of automated busses. In [103], iBus was introduced with a strong dual mode architecture. It goes through three processes similar to a human driver: perception, decision making and taking action but using software and hardware. This iBus was tested in China. Another automated bus in China is called an Alpha Bus [104]. Also, on 2015, a signed agreement was conducted for the trial of an automated shuttle service in Sentosa, Singapore as noted by [105] and by 2022, commuters in Singapore will ride the Smart bus in three districts Punggol, Tengah and the Jurong Innovation District (JID) [106]. Moreover, a self-driving Olli bus was introduced in the USA which has many features starting from moving people to their targeted destination to communication with the passenger about the journey, surrounding environment and about Olli itself [107].

Another highlight in the AI advancements of demand responsive public transportation is the flexible on-demand bus services which operate under flexible schedule and routes. These services aim to provide on delivering the door-to-door convenience of taxis at a fraction of its costs along with the efficiency of the bus model. There are many attempts and trails are happening around the world to improve the conventional bus service efficiency, and to provide convenient service to users. In order to empower transit operators to reduce delay and to provide an on-time service, Optibus has launched an AI-driven On-Time optimization solution [61,108]. China has introduced an advanced, personalized and flexible demand responsive public transport service called customized bus (CB) $[109,110]$. An intelligent flexible bus service called BRIDJ launched in 2014 in Boston, Massachusetts [111]. This uses real-time traffic big data and passenger inputs to evaluate how and 
to where the passengers want to move. This software is capable of finding the fastest route and only stops at locations as per passenger requests to optimise the service.

It is important to provide an efficient customer-centric service. Also, it is essential to achieve the operational cost effectiveness by considering the adequate fleet size and operate the optimum occupancy. For this reason, passenger demand prediction plays a key role in order to detect the bus headway, a fleet size that occupies the fleet in an optimum manner. Therefore, passenger demand prediction models developed by $[112,113]$ for a conventional bus system can be considered as a base study.

Another area where AI applications have demonstrated real value is in vehicle tracking on transport networks. An Automatic Vehicle Location (AVL) system has been introduced to improve operational efficiency of public transport, manage operational control and enhance overall quality of public transport services [114]. This system is able to extract data to track transportation units in real time by using GPS signals, detect problems to inform vehicles on any changes and manage alternative routes. Also, it provides information to passengers via APPS on their mobile devices. References [114,115] show a successful case study of AVL based GPS system implemented in Cagliari city in Italy. The AVL control centre receives real time data for the location of busses every $30 \mathrm{~s}$ these data are used for three purposes:

- Enhance the reliability of buses' services;

- Prioritise movement of buses at traffic signals; and

- Provide information to passengers about the schedule of the bus near bus stops.

Another example of AVL-based GPS systems is the iBus system in London as noted by [116]. Furthermore, to ensure a successful implementation of AVL systems, innovative AI-based data management system have also been proposed. According to [117], machine learning clustering techniques were interacted with AVL data to increase the performance of AVL system for planning bus schedules in Portugal. This integration helped to evaluate whether a change in a schedule will meet the network needs. In another study [118], the authors suggested finding patterns among AVL data using unsupervised clustering machine learning techniques to improve schedules for public transport in Sweden. Moreover, to improve the design of new routes and enhance bus scheduling, reference [119] use data collected from AVL in BusGrid system to predict future passenger demand on bus stops and routes using supervised machine learning techniques. The results demonstrated that the system can provide an accurate passenger demand prediction at bus stops. However, there are many challenges and research gaps related to AVL-based Public transport planning as stated by [120]. First, the absence of reliability evaluation for current Schedule Planning (SP) indicators by measuring the frequency in each route. In addition, assessment of whether SP can cover behaviour of the recent systems and meets the current demand pattern. The second gap is the evaluation of using ANN to enhance travel time prediction based regression problems and enhance the bus arrival time schedule using regression models and speed-based Kalman filters. The last challenge is how to make an automatic control strategy using AVL and Automatic Passengers Count APC data for fast response to events and effective public transport planning. In another study [121], the authors address the importance of using smart card data for the utilisation of public transport user's demand and behaviour. Most smart card data provide entry data for passengers only, hence, many research were conducted on estimating the destination of passengers using different methods from these data. One of these method is deep learning conducted by [121,122]. A comparison between methods is conducted based on quality of smart card data, Transit mode, sample size, data source, validation (quality of algorithms), sensitivity analysis and parameters affecting the proposed models. The authors discovered that most papers don't validate the results of their models and they don't carry a sensitivity analysis. Also, the authors suggested improving the current model by adding land-use factors and information relation to public transport networks. Multi sources of data can be considered and integrated to estimate the destination of the passenger such as: AVL, Video data and GPS data. 


\subsection{Intelligent Urban Mobility}

The future vision for intelligent urban mobility is smarter decision-making based on real-time information, and network optimization by efficient use of infrastructure. Importantly, fostering a better, safe, and healthier transportation system which creates an intelligent connectivity to achieve a sustainable, seamless and environmental friendly network. Recently, the world was at the cusp of emerging autonomous vehicles $(\mathrm{AV})$, that is, vehicles capable of moving without the support and guidance of a human driver [123].

\section{Autonomous Vehicles}

Autonomous Vehicles (AV) rely on AI software based on deep learning techniques. This approach works by teaching the vehicle how to drive while maintaining safe headways, lane discipline, and control etc. AVs are forecast to have a major change in how transportation systems are operated around the world and their impact on traffic safety and traffic congestions has been predicted in some detail, along with their potential to shift travel behaviour [124]. These AVs are expected to change the travel patterns of people to result in different social structures and urban forms. They will help in car sharing and ride sharing with new business models for solutions to existing barriers, such as limited accessibility and reliability [125].

In [126], the authors showed the history behind autonomous vehicles. It has been initiated long time ago in the late 1950s but it became a reality in 1968 when an "urbmobile" first electric vehicle was introduced by The Cornell Aeronautical Laboratory, but the technology at that time didn't encourage the usage of it. In recent years, the availability of sensors and cameras have increased the necessity to create more reliable and safer roads by fully automating the vehicles for the future vision of transportation. However, some researchers believe that it is challenging for the vehicle to acknowledge all the surrounding in urban suburbs, it needs a huge amount of data to recognize traffic signals, other vehicles, road signs etc., considering the weather and lightning [127]. Moreover, software challenges including the security and integrity of the system are considered a vital challenge to this emerging technology [62].

These autonomous vehicles mainly consist of two components and they are hardware and software architecture. The hardware architecture is composed of actuators, sensors and a computer system while the software component compromised of a navigation module, a localization algorithm and a perception to detect moving objects [128]. AVs are based on sensor-based technologies and communication across connected-vehicles. The advancement of this AV is fully automated vehicles, where the challenge is to enabling the vehicle to move safely, in between other vehicles on road avoiding obstacles, and pedestrians. This ability is developed with the knowledge of artificial intelligence, pattern recognition algorithms along with the use of sensors and 3D cameras [129].

In 2010, Google presented its Toyota Prius automated car in the United States. It is estimated to save over 30,000 lives and save the yearly costs related to road accidents to 270 billion in the USA. In addition, it will decrease the need for parking lots as the vehicle will park itself in remote areas. By 2012, Google introduced the Lexus RX450h and a fully automated vehicle with no steer has been introduced named "Firefly" in 2015. Now, according to [130], Google continues to develop its automated vehicle project through an independent company named "Waymo".

However, social acceptance is important to acknowledge as a challenge for customer acceptance as noted in [62]. A global study on a different aspect of what makes the cognitive intelligent vehicle more appealing to passengers has been conducted by [131]. These aspects were as follows:

- Self-healing: Vehicles can recognize the error with themselves and fix it.

- Self-socializing: The ability of a vehicle to interact with the surrounding infrastructure, other vehicles and humans in natural language.

- Self-learning: The vehicle utilizes its own behaviours, driver, occupants, and the surrounding environment. 
- Self-driving: The ability of the vehicle to drive itself, with some automated limitation in a controlled environment.

- Self-configuring: Each mobility contains digital information to identify the desired and personalized vehicle experience.

- Self-Integrating: The ability to integrate with other systems in the transport like any other intelligent transport devices.

According to the results of the study conducted by [131], passenger responses showed that people are more interested in self-healing (59\%) and self-socializing (55\%) than the self-driving technology itself ( $54 \%$ to self-driving).

Despite the challenges, there are many benefits that can arise from the usage of automated vehicles in the future such as, enhanced road safety $[108,111]$, reduced congestion and reduction in delays and travel times [132-134], reducing car ownership [135] and lowering emissions and increasing fuel efficiency $[62,136]$. These benefits make the autonomous mobility an economical and reliable technique to adopt for the future of transport.

\section{The Limitation of AI Techniques}

AI methods have initiated different criticism since they were introduced to the field of transportation. One of the major limitations to AI is considering ANNs as a "black box" [137]. This means that the relationship between the input and the output is developed without any knowledge to the internal computations of the system. Also, it was suspected of the ability of ANNs to generalize in cases where some information is missing in the data sets. However, research overcome this limitation by combining neural network with other traditional technique and other AI tools as a hybrid solution to fix this problem [138-140]. However, this requirement of hybridization to improve the performance especially in multi-scenarios is considered as a general weakness [54].

Development of an AI-based for an efficient transportation system is very complicated, due to the creation of a mechanical intelligence along with the proper understanding the human-based information [141]. Until today, AI applications in transportation are limited to specific ITS applications such as data analysis and predictions of future mobility. It would be more efficient if AI applications are capable of handling the full range of the process. Accordingly, realizing the full potential of AI to develop applications that are capable of operating as standalone systems is needed. Therefore, it is important to introduce AI knowledge in traffic analysis, data collection and storage, decision making and optimization modelling in future researches [142]. When AI techniques are based on data, which is collected from classical methods using loop detectors, sensors, and actuators etc. the accuracy and on-time predictions are not reliable. Therefore, departing from classical data collection methods to new AI-based technologies is important as that may provide data mining tools that are novel and easily deployable.

Another limitation is the ability to reach the finest optimum solution for the raster algorithms AI tools (e.g., GA and ACO) [143]. In addition, mathematical computations methods can provide a true understanding of the internal structure of the problem and the nature of the solution unlike using AI raster algorithms. However, in hard optimization problems where it is impossible to use traditional mathematical techniques, the quick analytics results generated from these algorithms are better than no solutions. Also, the research stated above proved that these algorithms reach a good solution in most cases. The parameters and assumptions need to be adjusted and rerun multiple times to reach the best solutions and have more insights into the problem. Another limitation is the bias introduced in the training data which in most cases is verified by humans who may introduce errors and biases in labelling.

In transportation, the capability to forecast short and long-term traffic flow is essential. The challenge is to forecast under unexpected events and adverse weather conditions. Unfortunately, the existing AI techniques are not capable of addressing such events and conditions. Therefore, the development of weather and incident responsive algorithms and prediction schemes is important 
to obtain high accuracy. The AI in developing these algorithms would enhance the efficiency in online computations and improve the standardizing the requirements with regard to spatial and temporal data coverage [142]. Most of the AI approaches such as NN in time series transport applications, rarely incorporate with any testing of the properties of the error and model specification, which is considered as a limitation. Therefore, for future research statistics and AI should have an interdependent relationship to improve specifically in terms of core model development and goodness of fit, and capability of analysing big data efficiently and accurately [142]. Apart from the above specific limitations of AI, there are few other general limitations. These include the high cost when developing smart technologies due to its complexity and ongoing maintenance, lack of customer privacy and transparency of AI-driven technologies.

Another major limitation, the computation complexity of $\mathrm{AI}$ is discussed in the next section.

\section{Computation Complexity of AI Algorithms}

An algorithm is a set of rules that target a specific problem to solve. The design and the analysis of Algorithms are an important part of AI techniques. It requires to be effective in terms of less time required to process the algorithm for a large number of input data. Also, sufficient small use of computer memory will determine the efficiency of an algorithm. The most known method to evaluate an algorithm is Big O notation [144,145]. It represents the upper bound on the number of operations a function would have to produce. The number of operations changes when extra data added, this number will help in identifying how long the process will take to complete the function i.e., it removes all the constants factors when the input size of data approaches to infinity. In an ideal scenario, when the number of data increases the number of operation stays constant When the input size. However, this cannot happen in a real-life scenario. In a simple algorithm scenario, the number of input data increases, the number of operations grows at a constant rate. While as, in difficult algorithm scenarios, the number of operations and computations are explained in polynomial time $\left(\mathrm{X}^{2}\right)$, exponential time $\left(2^{\mathrm{X}}\right)$ and factorial time (X!). According to [146] the theoretical explanation for algorithm complexity is expressed in a P problem and NP problem. These problems require fast computation computers to solve the complexity of the growing density of data. $\mathrm{P}$ problem can be verified by an algorithm that runs in polynomial time and solved using Deterministic Turning Machine as noted by [147] problem that required fast programs but the computation process is not very challenging and hard for large data sets compared to NP. NP is verified by Polynomial time algorithms and solved using Non-Deterministic Turning Machine [148]. The idea is to have many computers providing all possible solution for the same problem at the same time and giving the best solution at a polynomial time. NP is classified into NP-hard and NP-complete. The NP-hard problem exists when trying to develop an algorithm that works for all every input while NP-complete represents a problem that is impossible to solve in a polynomial time and hard to give an approximate solution in the optimization problem.

In transport, it includes problems such as vehicle routing and optimum scheduling for drivers and road users. The complexity of computation restricts $\mathrm{AI}$ techniques as most $\mathrm{AI}$ algorithms are classified as NP problem and NP-complete problem. In Deep learning, many hidden layers are constructed in the architecture of the network. Hence, complexity relies upon when big data includes noise and distortions that are hard extract features from. In transport, data can be collected from multiple sources ranging from sensors on the road, connected devices, toll gantries, GPS to Cloud applications, etc. these sources stores big data on different transportation features. Examples of those features are traffic flow, speed, and occupancy and traveler behavior. Hence, it complexes the computation process to solve a specific problem.

One solution to overcome this challenge is map-reduce which is part of HADOOP Framework. the computations are distributed in the framework into more than one computer to ease the process of analyzing big data [149]. The idea was first developed by [150]. The first training set of data is divided into multiple subsets and distributed to multiple parallel computers. Each computer will run part of 
the data set individually at the same time and then all the results will be combined at the end in a centralized master server. This will increase the computation running time. In this case, many learning algorithms will be equal to computation sum of functions of the divided training sets. Another solution, based on map-reduce can also be applied in one computer that has multiple core or CPUs. In which each CPU or core will process part of the large datasets to generate the best solution and speed up the process of AI learning algorithms [150]. Reference [151] shows a challenge in the fast computation for greedy algorithms as they are a sequential and applies the algorithms to a map-reduce parallel computation to increase its efficiency. Similarly [149], the authors present a map-reduce approach to solve and manage the complexity of dense traffic data. The authors proved it as a successful method to adapt when detecting anomalies of traffic events for the big file of data. This works by dividing these data to subsets and analyze each set for a parallel computation. While [152] use map-reduce to predict bus arrival time. They use Clustering K-Mean algorithm to divide the running time of a bus. K-Mean algorithm is capable of clustering the data into k groups. It runs multiple time to find more than one good configuration. Then, the authors use Back Propagation NN to predict the arrival time of the bus on a map-reduce platform. The results show a more accurate and fast prediction compared with a traditional Back Propagation method. Similar to that, reference [153] use map-reduce to predict traffic flow for a transport network in Russia. However, the authors use the K-Nearest Algorithm taking into account the spatial-temporal characteristic of flow. According to [154], this algorithm classifies input data based on a majority vote of its neighbors. The inputs are assigned to the most common class among its $\mathrm{K}$ nearest neighbors, the best $\mathrm{k}$ group choice depends on the observations and the noise of the data can be reduced with a larger value of $\mathrm{k}$ for the classification problem.

\section{Future of AI Is Governed by Deep Learning}

Deep learning innovation continuous to unfold the mystery behind the huge amount of data generated in different industries [155]. In 2016, according to [156] the size of the market for this technique was valued at 272 Million USD and it is expected to grow due to its high data storage capability, accurate computing power and its ability to manage the complexity within high volume data. This value was based on the application of deep learning in image recognition tasks for healthcare sectors and Facebook facial recognition features. Also, Automotive, financial and data mining sectors continue to improve their operations by adopting deep learning AI technology. In addition, recognizing pattern among data for a useful prediction for the future will lead to a rapid growth in the value of deep learning by 2025 to reach 10.2 USD billion [120].

According to [127], the author showed that the value of AI usage can be improved in terms of Business, economy, and society for Travel and transport sector. They noted that AI modern deep learning Neural Network can provide better value than the traditional techniques, see Figure 2.

As noted in Figure 2, the key areas where significant benefits can be derived is in the travel and transport-related services. For example, AI techniques can be used to find an optimum and fastest route for the convenient of road users and delivery service purposes. One European company has managed to detect real-time truck performance and driver behaviour by analysing information from sensors on the roads. This helped to reduce $15 \%$ of fuel cost and minimize delivery time. Also, Airlines can avoid the cost of cancelation by predicting the weather condition and congestion using AI techniques. Other benefits include: reduce traffic congestion, optimum public transport scheduling for the customer, safety of riders using self-driving vehicles and improve air quality. On average. The value increases from $62 \%$ to $128 \%$ for the travel sector and to $89 \%$ for transport and logistics. In the future, the application of autonomous vehicles will lead to the growth of deep learning values.

Furthermore, it is estimated that if $30 \%$ of vehicles were self-driving vehicles by 2030 , then the congestion cost will be decreased from 38 \$ billion to around 26 \$ billion in Australian cities [157] as shown in Figure 3. 


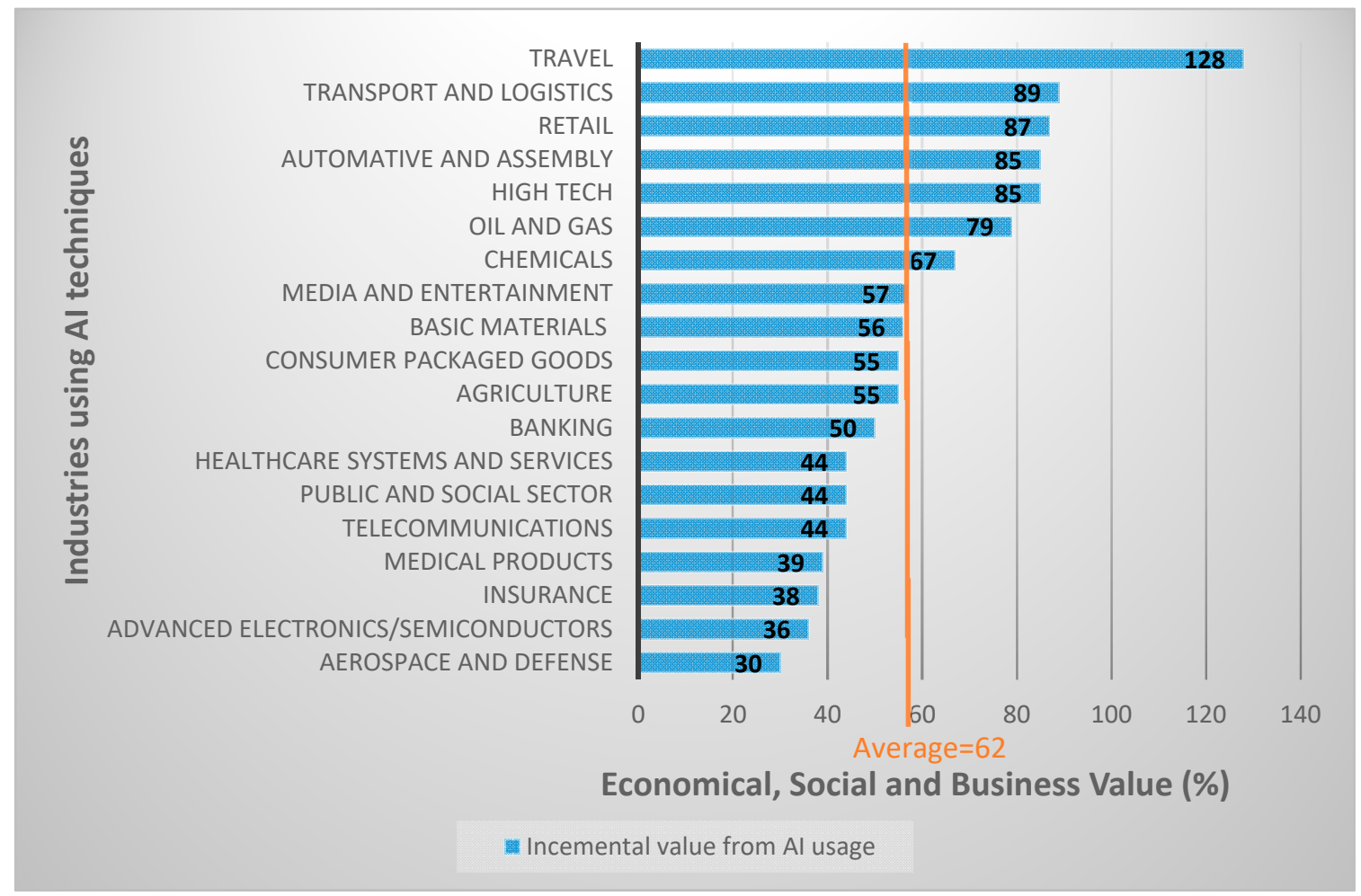

Figure 2. The performance improvement from AI-adapted from [127].

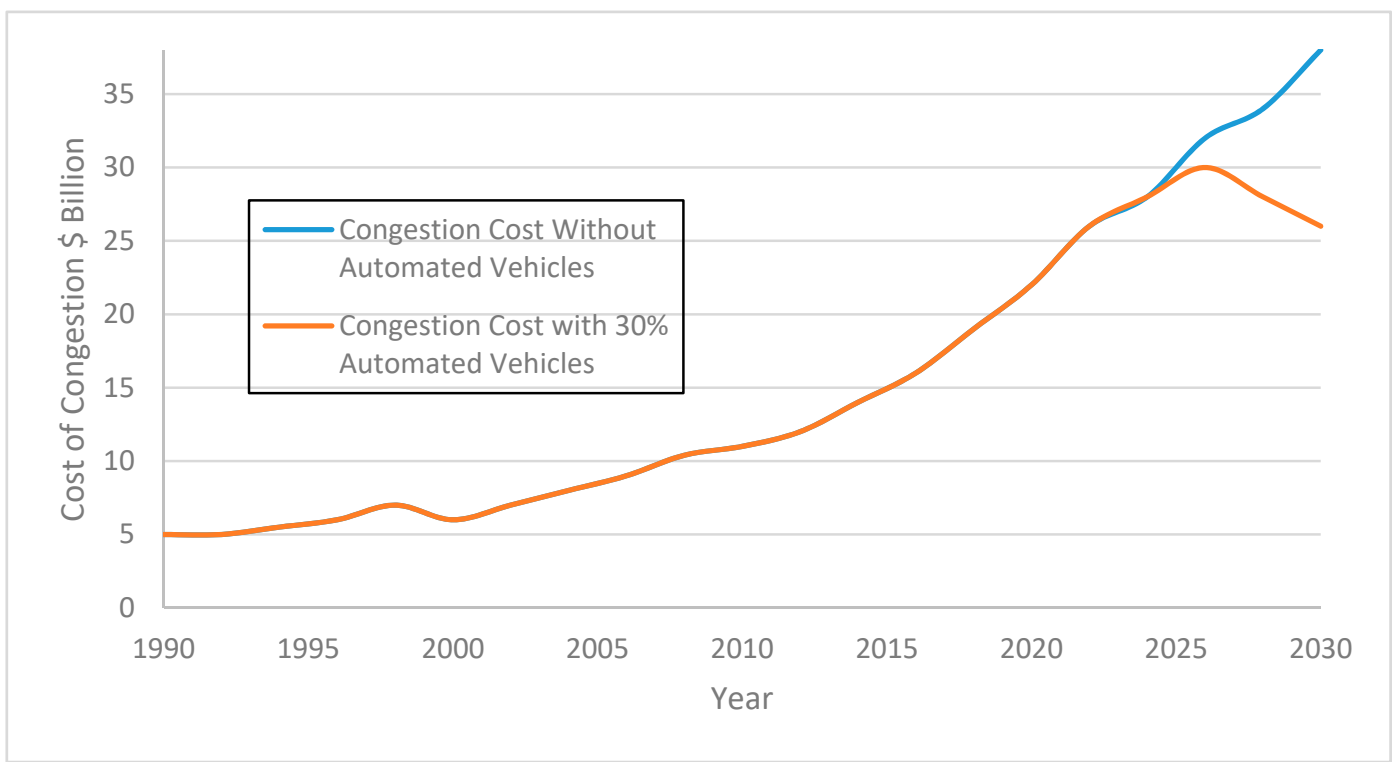

Figure 3. The effect of using $30 \%$ autonomous vehicles on road on congestion cost in Australia-adapted from [157].

\section{Future Research Work}

It has been noted that ANN is a robust model because it can cover multiple AI tasks and it doesn't need a deep understanding of the process for a certain targeted task. Its advantages also include relating inputs to outputs using pattern recognition. It also can manage a huge amount of data with well adjustment and performance when surrounded by noisy data. It saves time since it is a fast computation tool with good performance. It is successfully implemented when tested around different structures and different tasks. However, very few research has been conducted for a long-term traffic state prediction using deep learning architecture. Also, previous research focuses on only one or 
two traffic parameters to develop the model. Therefore, a future research will be directed towards enhancing predictive operations using more than two features and more than one hidden layer for the structure of the model.

The aim is to estimate future traffic based on historical and real-time information collected from detector stations located upstream and downstream and cameras attached on the freeway. A freeway in Melbourne/Australia will be selected as a Test Bed to demonstrate the feasibility of the research and the specific location and data gathering will be agreed on with the freeway authority at the early stage of the research. As seen in the previous research, the successful implementation of ANN is to choose the right architecture for the network i.e., the number of neurons in the hidden layers and the number of hidden layers. A deep learning system is chosen because it can detect increasingly subtle features of the input data (e.g., combinations of the on-ramp and mainline traffic flows, geometric bottlenecks and weather conditions) which together might produce unique traffic situations on the freeway. A structure of a multi-layered model is shown in Figure 4.

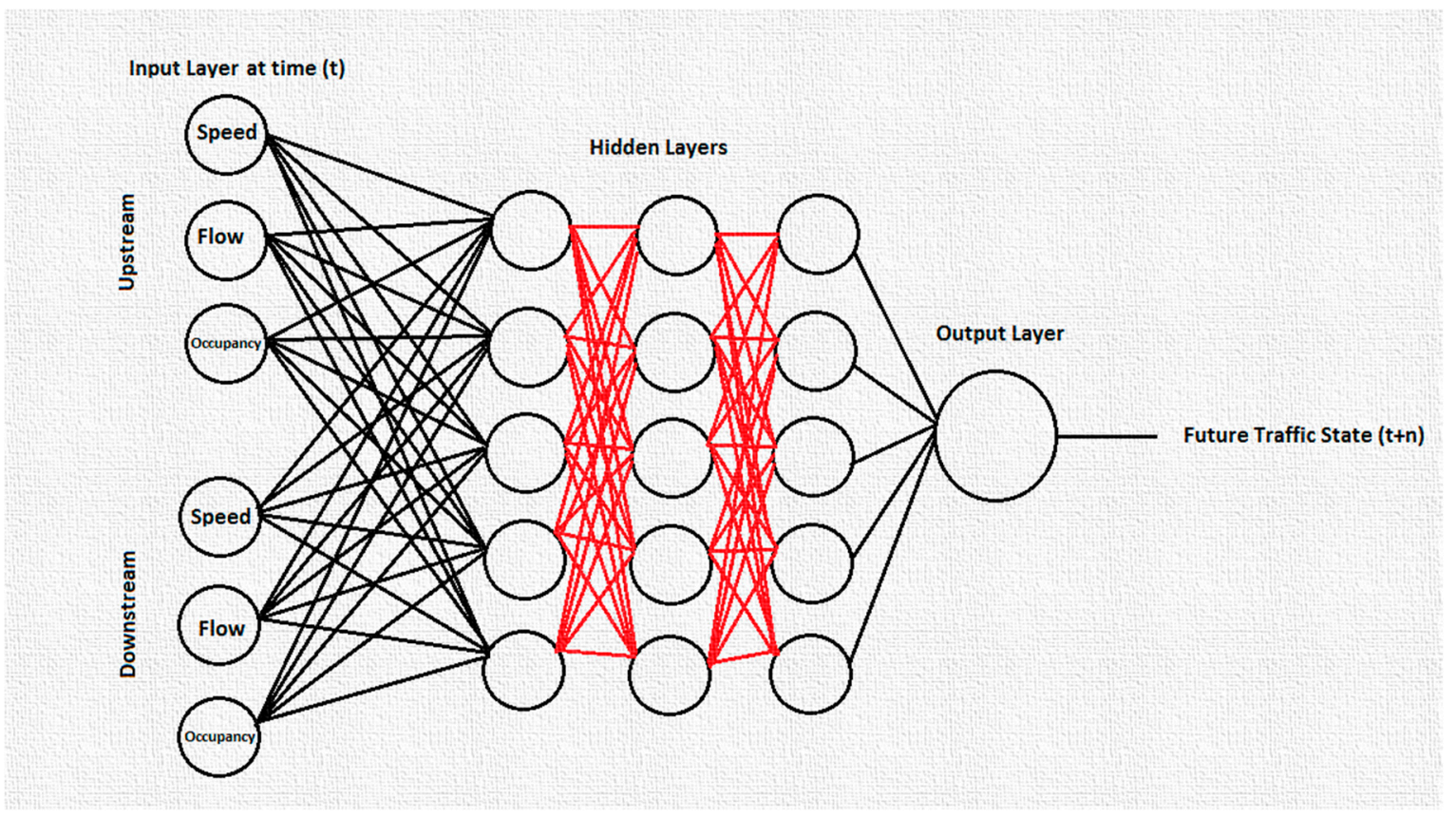

Figure 4. Deep learning system representation. Source: Authors.

The availability of data collected will define the type of model to develop. The input layer will include traffic-related data including speed, flow and occupancy and non-traffic related data such as weather conditions at the time $(t)$. The output represents the future traffic state $(t+n)$ where $n$ varies from 5 to $60 \mathrm{~min}$. It can be binary classification (congested and not-congested), categorical output (smooth flow, average flow, congested flow) and it can be presented as a numerical value ranging from 0 (not congested) to 1 (congested). The system will be developed using simulated data generated in AIMSUN Next 8.2.3 Software and validated using field data collected from a freeway in Melbourne. Then, it will be applied in several offline scenarios to test its performance. Other suggestion is to use the Recurrent Neural Network (RNN) or a combination of the Convolutional Neural Network (CNN) and RNN to model sensory data while using the CNN to model video data. Then, the two models of sensory and video data could be coupled to leverage the heterogeneous data for prediction. This is because of the spatial-temporal nature of the sensory data and the distinct properties between the data from sensors and cameras. This system will create smarter, safer and more productive journeys for customers, and increase the efficiency of the existing asset. Also, it will allow road authorities staff to anticipate network disruptions which would then help them to implement traffic management interventions and strategies to mitigate any adverse impacts before they occur on the freeway network. 


\section{Conclusions}

This paper presents an overview of the applications of AI to a variety of transport-related problems. The range of applications is expected to increase as our cities and transport systems become more instrumented providing much-needed data for AI application development. The review focused on a number of application areas which are expected to have more influence in future cities including autonomous vehicles, public transport, disruptive urban mobility, automated incident detection, future traffic status prediction, and traffic management and control. It shows that AI can be used to solve the challenge of increasing travel demand, $\mathrm{CO}_{2}$ emissions, safety concerns, and wasted fuels.

The literature abounds with case studies that show how $\mathrm{AI}$ is effective in designing and developing an optimal network for the community, finding an optimal schedule plans for public transport authorities, enhancing timing plans for traffic signals, and optimising routes for individual drivers. It is also applied for automated incident detection, detecting anomalies during flights and in image processors/video sequences for data collected from the roads. Moreover, in recent years, AI has been developed to use in traffic demand prediction, weather condition prediction, and future traffic state for management and control purposes and to alleviate congestion and fast decision making during hazardous situation i.e., road accidents. It can also help the authorities to make a decision on whether to add new infrastructures or expanding lanes, a decision on what route to take during an incident or severe weather conditions and decision on the amount of money required for maintenance and rehabilitation. Furthermore, automated vehicles and automated public transportation systems are also increasingly benefiting from AI tools to avoid disruptions, accidents, and congestion. Key limitations of AI were also addressed in particular the perception that neural networks are "black boxes" and also the issue surrounding introduction of bias in the training data as a result of having humans label the training examples.

Author Contributions: The authors' contributions are as follows: Conceptualisation, R.A. and H.D.; Methodology, R.A., H.D.; original draft preparation, R.A.—Writing, R.A., S.L.—Edit review and editing, R.A., S.L., S.A.B. and H.D.; supervision, H.D. and S.A.B.

Funding: This research received no external funding.

Acknowledgments: Rusul Abduljabbar acknowledges her Ph.D. scholarship provided by the Iraqi Government and Swinburne University of Technology. Sohani Liyanage acknowledges her Ph.D. scholarship provided by the Swinburne University of Technology.

Conflicts of Interest: The authors declare no conflict of interest.

\section{Abbreviations}

$\begin{array}{ll}\text { AI } & \text { Artificial Intelligence } \\ \text { ANN } & \text { Artificial Neural Network } \\ \text { AIS } & \text { Artificial Immune system } \\ \text { SA } & \text { Simulated Annealing } \\ \text { BCO } & \text { Bee Colony Optimization } \\ \text { GA } & \text { Genetic algorithms } \\ \text { RNN } & \text { Recurrent Neural Network } \\ \text { CNN } & \text { Convolutional Neural Network } \\ \text { PNN } & \text { Probabilistic Neural Network } \\ \text { ITS } & \text { Intelligent Transport Systems } \\ \text { NDP } & \text { Network Design Problem } \\ \text { DBN } & \text { Deep Belief Network } \\ \text { AV } & \text { Automated Vehicles }\end{array}$

\section{References}

1. Sadek, A. Artificial Intelligence in Transportation. Transp. Res. Circ. 2007, E-C113, 72-79.

2. Yegnanarayana, B. Artificial Neural Networks; PHI Learning Pvt. Ltd.: New Delhi, India, 1999; p. 476. 
3. Abraham, A. Artificial Neural Networks. Handbook of Measuring System Design; Sydenham, P.H., Thorn, R., Eds.; John Wiley \& Sons, Ltd.: Hoboken, NJ, USA, 2005.

4. Minsky, M.; Papert, S. Perceptron Expanded Edition; MIT Press: Cambridge, MA, USA, 1969.

5. Wolpert, D.H. Bayesian Backpropagation Over I-O Functions Rather Than Weights. Adv. Neural Inf. Process. Syst. 1993, 6, 200-207.

6. Parker, D. Learning Logic: Technical Report TR-87, Center for Computational Research in Economics and Management Science; The MIT Press: Cambridge, MA, USA, 1985.

7. Getoor, B.; Taskar, L. Introduction to Statistical Relational Learning; Volume L of Adaptive Computation and Machine Learning; MIT Press: Cambridge, MA, USA, 2007.

8. Bacciu, A.; Micheli, D.; Sperduti, A. Compositional generative mapping for tree-structured data-Part I: Bottom-up probabilistic modeling of trees. IEEE Trans. Neural Netw. Learn. Syst. 2012, 23, 1987-2002. [CrossRef] [PubMed]

9. Krizhevsky, A.; Sutskever, I.; Hinton, G.E. ImageNet Classification with Deep Convolutional Neural Networks. Adv. Neural Inf. Process. Syst. 2012, 60,1-9. Available online: https://papers.nips.cc/paper/ 4824-imagenet-classification-with-deep-convolutional-neural-networks.pdf (accessed on 27 December 2018). [CrossRef]

10. Kim, P. Convolutional Neural Network; MATLAB Deep Learning Apress: Berkeley, CA, USA, 2017.

11. McCann, M.T.; Jin, K.H.; Unser, M. Deep Convolutional Neural Network for Inverse Problems in Imaging. IEEE Signal Process. Mag. 2017, 34, 85-95. [CrossRef]

12. Zhang, X.Y.; Yin, F.; Zhang, Y.M.; Liu, C.L.; Bengio, Y. Drawing and Recognizing Chinese Characters with Recurrent Neural Network. IEEE Trans. Pattern Anal. Mach. Intell. 2018, 40, 849-862. [CrossRef] [PubMed]

13. Du, M.N.S.; Swamy, K.L. Recurrent Neural Networks. In Neural Networks and Statistical Learning; Springer: London, UK, 2014.

14. Caterini, D.E.; Chang, A.L. Recurrent Neural Networks. In Deep Neural Networks in a Mathematical Framework; Springer: Cham, Switzerland, 2018.

15. Patterson, D. Introduction to Artificial Intelligence and Expert Systems; Prentice-Hall, Inc.: Upper Saddle River, NJ, USA, 1990.

16. Australian Infrastructure Audit. Infrastructure Australia. Our Infrastructure Challenge. 2015. Available online: https://infrastructureaustralia.gov.au/policy-publications / publications/files/AustralianInfrastructure-Audit-Volume-1.pdf (accessed on 27 September 2018).

17. Linking Melbourne Authority. Linking Melbourne. Annual Report. Available online: https://www.parliament.vic.gov.au/file_uploads/Linking_Melbourne_Authority_Annual_Report_ 2014-2015_CwBGv8WN.pdf (accessed on 5 March 2018).

18. Klügl, F.; Bazzan, A.L.C.; Ossowski, S. Agents in traffic and transportation. Transp. Res. Part C Emerg. Technol. 2010, 18, 69-70. [CrossRef]

19. Doğan, E.; Akgüngör, A.P. Forecasting highway casualties under the effect of railway development policy in Turkey using artificial neural networks. Neural Comput. Appl. 2013, 22, 869-877.

20. Budalakoti, S.; Srivastava, A.N.; Otey, M.E. Anomaly detection and diagnosis algorithms for discrete symbol sequences with applications to airline safety. IEEE Trans. Syst. Man Cybern. Part C Appl. Rev. 2009, 39, 101-113. [CrossRef]

21. Akgüngör, A.P.; Doğan, E. An artificial intelligent approach to traffic accident estimation: Model development and application. Transport 2009, 24, 135-142.

22. Dia, H.; Rose, G. Development and evaluation of neural network freeway incident detection models using field data. Transp. Res. Part C Emerg. Technol. 1997, 5, 313-331. [CrossRef]

23. Wang, R.; Fan, S.; Work, D.B. Efficient multiple model particle filtering for joint traffic state estimation and incident detection. Transp. Res. Part C Emerg. Technol. 2016, 71, 521-537. [CrossRef]

24. Wang, R.; Work, D.B. Interactive multiple model ensemble Kalman filter for traffic estimation and incident detection. In Proceedings of the 17th International IEEE Conference on Intelligent Transportation Systems (ITSC), Qingdao, China, 8-11 October 2014; pp. 804-809.

25. Dia, H. An object-oriented neural network approach to short-term traffic forecasting. Eur. J. Oper. Res. 2001, 131, 253-261. [CrossRef]

26. Huang, W.; Song, G.; Hong, H.; Xie, K. Deep architecture for traffic flow prediction: Deep belief networks with multitask learning. IEEE Trans. Intell. Transp. Syst. 2014, 15, 2191-2201. [CrossRef] 
27. Jiang, H.; Zou, Y.; Zhang, S.; Tang, J.; Wang, Y. Short-Term Speed Prediction Using Remote Microwave Sensor Data: Machine Learning versus Statistical Model. Math. Probl. Eng. 2016, 2016, 9236156. [CrossRef]

28. Ledoux, C. An urban traffic flow model integrating neural networks. Transp. Res. Part C Emerg. Technol. 1997, 5, 287-300. [CrossRef]

29. Lv, Y.; Duan, Y.; Kang, W.; Li, Z.; Wang, F.Y. Traffic Flow Prediction with Big Data: A Deep Learning Approach. IEEE Trans. Intell. Transp. Syst. 2014, 16, 865-873. [CrossRef]

30. More, R.; Mugal, A.; Rajgure, S.; Adhao, R.B.; Pachghare, V.K. Road traffic prediction and congestion control using Artificial Neural Networks. In Proceedings of the 2016 International Conference on Computing, Analytics and Security Trends (CAST), Pune, India, 19-21 December 2016; pp. 52-57.

31. Wu, Y.; Tan, H.; Qin, L.; Ran, B.; Jiang, Z. A hybrid deep learning based traffic flow prediction method and its understanding. Transp. Res. Part C Emerg. Technol. 2018, 90, 166-180. [CrossRef]

32. Theofilatos, A.; Yannis, G.; Kopelias, P.; Papadimitriou, F. Predicting Road Accidents: A Rare-events Modeling Approach. Transp. Res. Procedia 2016, 14, 3399-3405. [CrossRef]

33. Król, A. The Application of the Artificial Intelligence Methods for Planning of the Development of the Transportation Network. Transp. Res. Procedia 2016, 14, 4532-4541. [CrossRef]

34. Xu, T.; Wei, H.; Wang, Z.D. Study on continuous network design problem using simulated annealing and genetic algorithm. Expert Syst. Appl. 2009, 36 Pt 2, 2735-2741. [CrossRef]

35. Ceylan, H.; Bell, M.G.H. Traffic signal timing optimisation based on genetic algorithm approach, including drivers' routing. Transp. Res. Part B Methodol. 2004, 38, 329-342. [CrossRef]

36. Ulusoy, Ü.; Sivrikaya-Şerifoğlu, G.; Bilge, F. A genetic algorithm approach to the simultaneous scheduling of machines and automated guided vehicles. Comput. Oper. Res. 1997, 24, 335-351. [CrossRef]

37. Karoonsoontawong, A.; Waller, S. Dynamic Continuous Network Design Problem: Linear Bilevel Programming and Metaheuristic Approaches. Transp. Res. Rec. J. Transp. Res. Board 2006, 1964, 104-117. [CrossRef]

38. Xu, T.; Wei, H.; Hu, G. Study on continuous network design problem using simulated annealing and genetic algorithm. Expert Syst. Appl. 2009, 36 Pt 1, 1322-1328. [CrossRef]

39. Kirkpatrick, S.; Gelatt, C.D.; Vecchi, M.P. Optimization by Simulated Annealing. Science 1983, 220, 671-680. [CrossRef] [PubMed]

40. Yaseen, S.G.; L-Slamy, N.A. Ant Colony Optimization. Int. J. Comput. Sci. Netw. Secur. 2008, 8, 351-357.

41. Dorigo, M.; Gambardella, L.M.; Birattari, M.; Martinoli, A.; Poli, R.; Stützle, T. LNCS 4150—Ant Colony Optimization and Swarm Intelligence. In Proceedings of the 6th International Conference (ANTS 2008), Brussels, Belgium, 22-24 September 2008.

42. Timmis, J.; Neal, M.; Hunt, J. An artificial immune system for data analysis. Biosystems 2000, 55, $143-150$. [CrossRef]

43. Dasgupta, D.; Ji, Z. Artificial immune system (AIS) research in the last five years. In Proceedings of the 2003 Congress on Evolutionary Computation (CEC '03), Canberra, Australia, 8-12 December 2003; Volume 1, pp. 123-130.

44. Lučić, D.B.; Teodorović, P. Bee system: Modeling combinatorial optimization transportation engineering problems by swarm intelligence. In Proceedings of the TRISTAN IV Triennial Symposium on Transportation Analysis, Azores, Portugal, 13-19 June 2001.

45. Lucic, D.; Teodorovic, P. Transportation modeling: An artificial life approach. In Proceedings of the 14th IEEE International Conference on Tools with Artificial Intelligence (ICTAI 2002), Washington, DC, USA, 4-6 November 2002.

46. Lučić, D.; Teodorović, P. Computing with bees: Attacking complex transportation engineering problems. Int. J. Artif. Intell. Tools 2003, 12, 375-394. [CrossRef]

47. Lučić, D.; Teodorović, P. Vehicle routing problem with uncertain demand at nodes: The bee system and fuzzy logic approach. In Fuzzy Sets Based Heuristics for Optimization; Springer: Berlin/Heidelberg, Germany, 2003; pp. 67-82. 
48. Kahraman, C. Fuzzy Multi-Criteria Decision Making: Theory and Applications with Recent Developments (Vol. 16). Springer Science \& Business Media, 2008. Available online: https://books.google.com.au/ books?hl=en\&lr=\&id=s2GOmBVdXYoC\&oi=fnd\&pg=PR5\&dq=Fuzzy+multi-criteria+decision+making: theory+and+applications+with+recent+\&ots=cMJfePwACg\&sig=VeZcfHidR_cvK49vIcmi5VvoE_w\# $\mathrm{v}=$ onepage\&q=Fuzzy\%20multi-criteria\%20decision\%20making\%3Atheory\%20and\%20applications\% 20with\%20recent\&f=false (accessed on 18 March 2018).

49. Voracek, J. Prediction of mechanical properties of cast irons. Appl. Soft Comput. 2001, 1, 119-125. [CrossRef]

50. Guarino, E.R.S.L.; Pfautz, J.D.; Cox, Z. Modeling human reasoning about meta-information. Int. J. Approx. Reason. 2009, 50, 437-449. [CrossRef]

51. Kulak, C.K.O.; Durmusoglu, M.B. Fuzzy multi-attribute equipment selection based on information axiom. J. Mater. Process. Technol. 2005, 169, 337-345. [CrossRef]

52. Qureshi, M.F.; Shah, S.M.A.; Al-Matroushi, G.I.G. A Comparative Analysis of Multi-Criteria Road Network. Eur. Cent. Res. Train. Dev. UK 2013, 27-47. Available online: http://www.eajournals.org/wp-content/ uploads / A-Comparative-Analysis-of-Multi-criteria-Road-Network.pdf (accessed on 5 July 2018).

53. Murat, N.U.Y. Route choice modelling in urban transportation networks using fuzzy logic and logistic regression methods. J. Sci. Ind. Res. 2008, 67, 19-27.

54. Harcourt, P. Route Optimization Techniques: An Overview. Available online: https://www.ijser.org/ researchpaper/ROUTE-OPTIMIZATION-TECHNIQUES-AN-OVERVIEW.pdf (accessed on 11 September 2018).

55. Aretakis, N.; Roumeliotis, I.; Alexiou, A.; Romesis, C.; Mathioudakis, K. Turbofan Engine Health Assessment from Flight Data. J. Eng. Gas Turbines Power 2014, 137, 041203. [CrossRef]

56. Bagloee, M.; Sarvi, S.A.; Patriksson, M. A hybrid branch-and-bound and benders decomposition algorithm for the network design problem. Comput. Civ. Infrastruct. Eng. 2017, 32, 319-343. [CrossRef]

57. Rodrigue, J.P. Parallel modelling and neural networks: An overview for transportation/land use systems. Transp. Res. Part C Emerg. Technol. 1997, 5, 259-271. [CrossRef]

58. Li, X.; Shi, X.; He, J.; Liu, X. Coupling simulation and optimization to solve planning problems in a fast-developing area. Ann. Assoc. Am. Geogr. 2011, 101, 1032-1048. [CrossRef]

59. Wen, Y.; Li, S.Y. Fastest Complete Vehicle Routing Problem Using Learning Multiple Ant Colony Algorithm. Adv. Mater. Res. Trans. Tech. Publ. 2011, 217, 1044-1049. [CrossRef]

60. Such, F.P.; Madhavan, V.; Conti, E.; Lehman, J.; Stanley, K.O.; Clune, J. Deep Neuroevolution: Genetic Algorithms Are a Competitive Alternative for Training Deep Neural Networks for Reinforcement Learning. arXiv 2017, arXiv:1712.06567.

61. Optibus \& Metropoline. Schedule Optimization Achieves Much More Than Just Cost Reduction. Available online: https:/ /www.optibus.com/case/company-name-5/ (accessed on 11 November 2018).

62. Bagloee, S.A.; Tavana, M.; Asadi, M.; Oliver, T. Autonomous vehicles: Challenges, opportunities, and future implications for transportation policies. J. Mod. Transp. 2016, 24, 284-303. [CrossRef]

63. Bell, J.E.; McMullen, P.R. Ant colony optimization techniques for the vehicle routing problem. Adv. Eng. Inform. 2004, 18, 41-48. [CrossRef]

64. Fries, R.; Chowdhury, M.; Brummond, J. Transportation Infrastructure Security Utilizing Intelligent Transportation Systems; John Wiley \& Sons: Hoboken, NJ, USA, 2008.

65. Nuzzolo, A.; Comi, A. Advanced public transport and intelligent transport systems: New modelling challenges. Transp. A Transp. Sci. 2016, 12, 674-699. [CrossRef]

66. Liu, X. Deep Reinforcement Learning for Intelligent Transportation Systems; No. Nips. arXiv 2018, arXiv:1812.00979.

67. Ferdowsi, A.; Challita, U.; Saad, W. Deep Learning for Reliable Mobile Edge Analytics in Intelligent Transportation Systems. arXiv 2017, arXiv:1712.04135.

68. Wang, C.; Li, X.; Zhou, X.; Wang, A.; Nedjah, N. Soft computing in big data intelligent transportation systems. Appl. Soft Comput. J. 2016, 38, 1099-1108. [CrossRef]

69. Gilmore, N.; Abe, J.F. Neural network models for traffic control and congestion prediction. J. Intell. Transp. Syst. 1995, 2, 231-252. [CrossRef]

70. Nakatsuji, T.; Kaku, T. Development of a Self-Organizing Traffic Control System Using Neural Network Models. Transp. Res. Rec. 1991, 1324, 137-145.

71. Choy, M.C.; Srinivasan, D.; Cheu, R.L. Cooperative, hybrid agent architecture for real-time traffic signal control. IEEE Trans. Syst. Man Cybern. Part A Syst. Hum. 2003, 33, 597-607. [CrossRef] 
72. Choy, M.; Cheu, R.; Srinivasan, D.; Logi, F. Real-time coordinated signal control through use of agents with online reinforcement learning. Transp. Res. Rec. J. Transp. Res. Board 2003, 1836, 64-75. [CrossRef]

73. Stojmenovic, M. Real time machine learning based car detection in images with fast training. Mach. Vis. Appl. 2006, 17, 163-172. [CrossRef]

74. Gu, F.; Qian, Y.; Chen, Z. From Twitter to detector: Real-time traffic incident detection using social media data. Transp. Res. Part C Emerg. Technol. 2016, 67, 321-342. [CrossRef]

75. Mahamuni, A. Internet of Things, machine learning, and artificial intelligence in the modern supply chain and transportation. Def. Transp. J. 2018, 74, 14. Available online: https: / / insights.samsung.com/2018/05/22/internet-of-things-machine-learning-and-artificial-intelligence-inthe-modern-supply-chain-and-transportation/ (accessed on 27 December 2018).

76. Wysocki, M.; Czuk, A. Jordan neural network for modelling and predictive control of dynamic systems. In Proceedings of the 2015 20th International Conference on Methods and Models in Automation and Robotics (MMAR), Miedzyzdroje, Poland, 24-27 August 2015; Volume 2, pp. 145-150.

77. Dong, J.; Shao, C.J.; Xiong, C.F.; Li, Z.H. Short-term traffic flow forecasting of road network based on Elman neural network. J. Transp. Syst. Eng. Inf. Technol. 2010, 1, 022.

78. ATOS 2012. Expecting the Unexptected Business Pattern Management. Atos Scientific Community. Available online: https:/ / atos.net/content/dam/global/ascent-whitepapers/ascent-whitepaper-expectingthe-unexpected-business-pattern-management.pdf (accessed on 2 October 2018).

79. Levene, C.; Litman, J.; Schillinger, S.; Toomey, I. How Advanced Analytics Can Benefit Infrastructure Capital Planning; Mckinsey Co. (Capital Proj. Infrastructure): New York, NY, USA, 2018.

80. Held, K.; Küng, M.; Çabukoglu, L.; Pareschi, E.; Georges, G.; Boulouchos, G. Future mobility demand estimation based on sociodemographic information: A data-driven approach using machine learning algorithms. In Proceedings of the 18th Swiss Transport Research Conference (STRC 2018), Ascona, Switzerland, 16-18 May 2018.

81. Yao, H.; Wu, F.; Jia, Y.; Lu, S.; Gong, P.; Ye, J. Deep Multi-View Spatial-Temporal Network for Taxi Demand Prediction. arXiv 2018, arXiv:1802.08714.

82. Mukai, N.; Yoden, N. Taxi demand forecasting based on taxi probe data by neural network. In Intelligent Interactive Multimedia: Systems and Service; Springer: Berlin/Heidelberg, Germany, 2012; pp. 589-597.

83. Li, B.; Zhang, D.; Sun, L.; Chen, C.; Li, S.; Qi, G.; Yang, Q. Hunting or Waiting? Discovering Passenger-Finding Strategies from a Large-Scale Real-World Taxi Dataset. In Proceedings of the 2011 IEEE International Conference on Pervasive Computing and Communications Workshops (PERCOM Workshops), Seattle, WA, USA, 21-25 March 2011; pp. 63-68.

84. Liao, J.; Zhou, S.; Di, L.; Yuan, X.; Xiong, B. Large-scale short-term urban taxi demand forecasting using deep learning. In Proceedings of the 23rd Asia and South Pacific Design Automation Conference, Jeju, Korea, 22-25 January 2018; pp. 428-433.

85. Ren, H.; Song, Y.; Wang, J.; Hu, Y.; Lei, J. A Deep Learning Approach to the Citywide Traffic Accident Risk Prediction. In Proceedings of the 2018 21st International Conference on Intelligent Transportation Systems (ITSC), Maui, HI, USA, 4-7 November 2018.

86. Chen, Q.; Song, X.; Yamada, H.; Shibasaki, R. Learning Deep Representation from Big and Heterogeneous Data for Traffic Accident Inference. In Proceedings of the Thirtieth AAAI Conference on Artificial Intelligence, Phoenix, AZ, USA, 12-17 February 2016; pp. 338-344.

87. Vasavi, S. Extracting Hidden Patterns Within Road Accident Data Using Machine Learning Techniques. In Information and Communication Technology; Springer: Singapore, 2018; pp. 13-22.

88. Taamneh, M.; Alkheder, S.; Taamneh, S. Data-mining techniques for traffic accident modeling and prediction in the United Arab Emirates. J. Transp. Saf. Secur. 2017, 9, 146-166. [CrossRef]

89. Oza, N.; Castle, J.P.; Stutz, J. Classification of aeronautics system health and safety documents. IEEE Trans. Syst. Man Cybern. Part C Appl. Rev. 2009, 39, 670-680. [CrossRef]

90. Williams, J.K. Using random forests to diagnose aviation turbulence. Mach. Learn. 2014, 95, 51-70. [CrossRef]

91. Loboda, I. Neural Networks for Gas Turbine Diagnosis; Machine Learning; Springer: New York, NY, USA, 2016; Chapter 8.

92. Laurell, C.; Sandström, C. The sharing economy in social media: Analyzing tensions between market and non-market logics. Technol. Forecast. Soc. Chang. 2017, 125, 58-65. [CrossRef] 
93. Geissinger, A.; Laurell, C.; Sandström, C. Technological Forecasting \& Social Change Digital Disruption beyond Uber and Airbnb-Tracking the long tail of the sharing economy. Technol. Forecast. Soc. Chang. 2018, in press. [CrossRef]

94. Firnkorn, J.; Müller, M. What will be the environmental effects of new free-floating car-sharing systems? The case of car2go in Ulm. Ecol. Econ. 2011, 70, 1519-1528. [CrossRef]

95. Cohen, B.; Kietzmann, J. Ride On! Mobility Business Models for the Sharing Economy. Organ. Environ. 2014, 27, 279-296. [CrossRef]

96. Inside BigData. AI in Sharing Economy. 2018. Available online: https://insidebigdata.com/2018/08/02/aisharing-economy/ (accessed on 14 September 2018).

97. Raymond, R.; Sugiura, T.; Tsubouchi, K. Location recommendation based on location history and spatio-temporal correlations for an on-demand bus system. In Proceedings of the 19th ACM SIGSPATIAL International Conference on Advances in Geographic Information Systems, Chicago, IL, USA, 1-4 November 2011; p. 377.

98. Mukai, J.; Watanabe, N.; Feng, T. Route Optimization Using Q-Learning for On-Demand Bus Systems. In Knowledge-Based and Intelligent Information and Engineering Systems; Springer: Berlin/Heidelberg, Germany, 2008; pp. 567-574.

99. Ma, J.; Song, C.; Ceder, A.; Liu, T.; Guan, W. Fairness in optimizing bus-crew scheduling process. PLoS ONE 2017, 12, e0187623. [CrossRef]

100. Hu, J.; Yang, Z.; Jian, F. Study on the optimization methods of transit network based on Ant Algorithm. In Proceedings of the IEEE International Vehicle Electronics Conference 2001, Tottori, Japan, 25-28 September 2001; pp. 215-219.

101. Chien, S.I.-J.; Ding, Y.; Wei, C. Dynamic Bus Arrival Time Prediction with Artificial Neural Networks. J. Transp. Eng. 2002, 128, 429-438. [CrossRef]

102. Jeong, R.; Rilett, R. Bus arrival time prediction using artificial neural network model. In Proceedings of the 7th International IEEE Conference on Intelligent Transportation Systems, Washington, DC, USA, 3-6 October 2004; pp. 988-993.

103. Han, W.; Zhang, X.; Yin, J.; Li, Y.; Li, D. Architecture of iBus: A Self-Driving Bus for Public Roads. Available online: https://www.sae.org/publications/technical-papers/content/2017-01-0067/ (accessed on 27 December 2018).

104. ChinaDaily. Self-Driving Bus Goes on World's First Trial Run on Public Road. Available online: http:/ / www.chinadaily.com.cn/china/2017-12/03/content_35179951.htm (accessed on 12 March 2018).

105. Huiling, B.; Goh, E. AI, Robotics and Mobility as a Service: The Case of Singapore. Field Actions Sci. Rep. J. Field Actions Spec. Issue 2017, 26-29. Available online: https:/ /journals.openedition.org/factsreports/4411 (accessed on 27 December 2018).

106. Yu, E. Singapore Aims to Drive up Standards for Autonomous Vehicles with Test Centre. Available online: https:/ / www.zdnet.com/article/singapore-aims-to-drive-up-standards-for-autonomousvehicles-with-test-centre/ (accessed on 23 March 2017).

107. Bausch, J. Local Motors and IBM Pave the Way for the Future of Automobiles. AspenCore IBM. Available online: https://www.electronicproducts.com/Internet_of_Things/Research/Local_Motors_and_ IBM_Pave_the_Way_for_the_Future_of_Automobiles.aspx (accessed on 16 April 2018).

108. Rosin, J. Optibus Uses Artificial Intelligence to Improve Mass Transit's On-Time Performance and Prevent Delays. 2018. Available online: https:// finance.yahoo.com/news/optibus-uses-artificial-intelligenceimprove-110000554.html?guccounter=1 (accessed on 13 April 2018).

109. Liu, T.; Ceder, A. Analysis of a new public-transport-service concept: Customized bus in China. Transp. Policy 2015, 39, 63-76. [CrossRef]

110. Ma, J.; Yang, Y.; Guan, W.; Wang, F.; Liu, T.; Tu, W.; Song, C. Large-scale demand driven design of a customized bus network: A methodological framework and Beijing case study. J. Adv. Transp. 2017, 2017, 3865701. [CrossRef]

111. Bridj. 2018. Available online: https://www.bridj.com/journey/ (accessed on 15 July 2018).

112. Zhou, C.; Dai, P.; Li, R. The passenger demand prediction model on bus networks. In Proceedings of the 2013 IEEE 13th International Conference on Data Mining Workshops, Dallas, TX, USA, 7-10 December 2013; pp. 1069-1076. 
113. Zhou, C.; Dai, P.; Zhang, Z. Passenger demand prediction on bus services. In Proceedings of the International of the Conference on Green Computing and Internet Things, ICGCIoT 2015, Delhi, India, 8-10 October 2016; pp. 1430-1435.

114. Barabino, B.; Di Francesco, M.; Mozzoni, S. Rethinking bus punctuality by integrating Automatic Vehicle Location data and passenger patterns. Transp. Res. Part A Pol. Pract. 2015, 75, 84-95. [CrossRef]

115. Tilocca, P.; Farris, S.; Angius, S.; Argiolas, R.; Obino, A.; Secchi, S.; Mozzoni, S.; Barabino, B. Managing Data and Rethinking Applications in an Innovative Managing Data and Rethinking Applications in an Innovative Mid-sized Bus Fleet Bus Fleet. Transp. Res. Procedia 2017, 25, 1899-1919. [CrossRef]

116. Hounsell, N.B.; Shrestha, B.P.; Wong, A. Data management and applications in a world-leading bus fleet. Transp. Res. Part C 2012, 22, 76-87. [CrossRef]

117. Mendes-moreira, J.; Moreira-matias, L.; Gama, J.; Freire, J.; Sousa, D. Validating the coverage of bus schedules: A Machine Learning approach. Inf. Sci. 2015, 293, 299-313. [CrossRef]

118. Khiari, O.; Moreira-Matias, J.; Cerqueira, L.; Cats, V. Automated setting of Bus schedule coverage using unsupervised machine learning. In Advances in Knowledge Discovery and Data Mining; Springer: Cham, Switzerland, 2016; pp. 552-564.

119. Samaras, I.; Fachantidis, P.; Tsoumakas, A.; Vlahavas, G. A prediction model of passenger demand using AVL and APC data from a bus fleet. In Proceedings of the 19th Panhellenic Conference on Informatics, Athens, Greece, 1-3 October 2015; pp. 129-134.

120. Moreira-Matias, L.; Mendes-Moreira, J.; de Sousa, J.F.; Gama, J. Improving Mass Transit Operations by Using AVL-Based Systems: A Survey. IEEE Trans. Intell. Transp. Syst. 2015, 16, 1636-1653. [CrossRef]

121. Li, T.; Sun, D.; Jing, P.; Yang, K. Smart card data mining of public transport destination: A literature review. Information 2018, 9, 18. [CrossRef]

122. Jung, J.; Sohn, K. Deep-learning architecture to forecast destinations of bus passengers from entry-only smart-card data. IET Intell. Transp. Syst. 2017, 11, 334-339. [CrossRef]

123. Zhang, W.; Guhathakurta, S.; Fang, J.; Zhang, G. Exploring the impact of shared autonomous vehicles on urban parking demand: An agent-based simulation approach. Sustain. Cities Soc. 2015, 19, 34-45. [CrossRef]

124. Fagnant, D.J.; Kockelman, K.M. The travel and environmental implications of shared autonomous vehicles, using agent-based model scenarios. Transp. Res. Part C Emerg. Technol. 2014, 40, 1-13. [CrossRef]

125. Kornhauser, A.L. Uncongested Mobility for All: New Jersey's Area-wide aTaxi System; Princeton University: Princeton, NJ, USA, 2013.

126. Poczter, L.; Jankovic, S.L. The Google Car: Driving toward a better future? J. Bus. Case Stud. 2014, 10, 7. [CrossRef]

127. McKinsey Global Institute. Notes from the AI Frontier. Insights from Hundreds of Use Cases; McKinsey Global Institute: San Francisco, CA, USA, 2018; p. 36.

128. Chong, Z.J.; Qin, B.; Bandyopadhyay, T.; Wongpiromsarn, T.; Rebsamen, B.; Dai, P.; Rankin, E.S.; Ang, M.H. Autonomy for Mobility on Demand; Springer: Berlin/Heidelberg, Germany, 2013; Volume 127.

129. Manyika, J.; Chui, M.; Bughin, J.; Dobbs, R.; Bisson, P.; Marrs, A. Disruptive Technologies: Advances That Will Transform Life, Business, and the Global Economy; McKinsey Global Insitute: San Francisco, CA, USA, 2013; p. 163.

130. Waymo. Available online: https:/ / waymo.com/tech/ (accessed on 24 May 2018).

131. Stanley, B.; Gyimesi, K. A New Relationship-People and Cars; IBM Institute for Business Value: Armonk, NY, USA, 2016; p. 21.

132. Anderson, J.; Kalra, N.; Stanley, K.; Sorensen, P.; Samaras, C.; Oluwatola, O. Autonomous Vehicle Technology: A Guide for Policymakers; RAND Corporation: Arlington, VA, USA, 2016.

133. Dresner, K.; Stone, P. Sharing the road: Autonomous vehicles meet human drivers. In Proceedings of the 20th International Joint Conference on Artificial Intelligence (IJCAI), Hyderabad, India, 6-12 January 2007; pp. 1263-1268.

134. Fajardo, D.; Au, T.-C.; Waller, S.; Stone, P.; Yang, D. Automated Intersection Control. Transp. Res. Rec. J. Transp. Res. Board 2011, 2259, 223-232. [CrossRef]

135. Fagnant, D.J.; Kockelman, K. Preparing a nation for autonomous vehicles: Opportunities, barriers and policy recommendations. Transp. Res. Part A Policy Pract. 2015, 77, 167-181. [CrossRef]

136. Hübner, H.P. Automated Driving-Where Are We Heading? Available online: https:/ /link.springer.com/ chapter/10.1007\%2F978-3-658-05978-1_5 (accessed on 30 March 2018). 
137. Olden, J.D.; Jackson, D.A. Illuminating the 'black box': A randomization approach for understanding variable contributions in artificial neural networks. Ecol. Model. 2002, 154, 135-150. [CrossRef]

138. Kanungo, D.P.; Arora, M.K.; Sarkar, S.; Gupta, R.P. A comparative study of conventional, ANN black box, fuzzy and combined neural and fuzzy weighting procedures for landslide susceptibility zonation in Darjeeling Himalayas. Eng. Geol. 2006, 85, 347-366. [CrossRef]

139. Setiono, J.Y.; Leow, R.; Thong, W.K. Opening the neural network black box: An algorithm for extracting rules from function approximating artificial neural networks. In Proceedings of the Twenty First International Conference on Information Systems, Brisbane, Australia, 10-13 December 2000; pp. 176-186.

140. Dayhoff, J.E.; DeLeo, J.M. Artificial neural networks: Opening the black box. Cancer 2001, 91, 1615-1635. [CrossRef]

141. Waltz, D.L. Artificial Intelligence: Realizing the Ultimate Promises of Computing in Computing Research. AI Mag. 1997, 18, 49-52.

142. Vlahogianni, E.I.; Karlaftis, M.G.; Golias, J.C. Short-term traffic forecasting: Where we are and where we're going. Transp. Res. Part C 2014, 43, 3-19. [CrossRef]

143. Chowdhury, A.W.; Sadek, M. Advantages and Limitations of Artificial Intelligence. Available online: https:/ /www.researchgate.net/profile/Said_Easa/publication/273576102_Design_and_construction_ of_transportation_infrastructure_httponlinepubstrborgonlinepubscircularsec168pdf/links / 55097a910cf26ff55f85932b.pdf\#page=14 (accessed on 10 June 2018).

144. Black, P. Big-O Notation. Dict. Algorithms Data Structures. Available online: http:/ /www.nist.gov / dads / HTML/bigOnotation.html (accessed on 20 July 2018).

145. Danziger, Big O Notation. 2010. Available online: http://www.scs.ryerson.ca/ \{\}mth110/Handouts/PD/ bigO.Pdf (accessed on 14 November 2018).

146. Fortnow, L. The status of the P versus NP problem. Commun. ACM 2009, 52, 78-86. [CrossRef]

147. Cook, S. The P Versus NP Problem. In The Millennium Prize Problems; American Mathematical Society: Providence, RI, USA, 2006; p. 86.

148. Feynman, R. Feynman Lectures on Computation; CRC Press: Boca Raton, FL, USA, 2018.

149. Adoni, A.H.; Nahhal, W.Y.; Aghezzaf, T.; Elbyed, B. The MapReduce-based approach to improve vehicle controls on big traffic events. In Proceedings of the 2017 International Colloquium on Logistics and Supply Chain Management (LOGISTIQUA), Rabat, Morocco, 27-28 April 2017.

150. Dean, S.; Ghemawat, J. MapReduce: Simplified data processing on large clusters. Commun. ACM 2008, 51, 107-113. [CrossRef]

151. Kumar, A.; Moseley, R.; Vassilvitskii, B.; Vattani, S. Fast greedy algorithms in mapreduce and streaming. ACM Trans. Parallel Comput. (TOPC) 2015, 2, 14. [CrossRef]

152. Zhang, S.; Gu, J.; Guan, J.; Zhang, L. Method of predicting bus arrival time based on MapReduce combining clustering with neural network. In Proceedings of the 2017 IEEE 2nd International Conference on Big Data Analysis (ICBDA), Beijing, China, 10-12 March 2017; pp. 296-302.

153. Agafonov, A.; Yumaganov, A. Spatial-Temporal K Nearest Neighbors Model on MapReduce for Traffic Flow Prediction. In International Conference on Intelligent Data Engineering and Automated Learning; Springer: Cham, Switzerland, 2018; pp. 253-260.

154. Larose, C.; Larose, D.T. Discovering Knowledge in Data: An Introduction to Data Mining; John Wiley Sons: Hoboken, NJ, USA, 2014.

155. Schmidhuber, J. Deep Learning in neural networks: An overview. Neural Netw. 2015, 61, 85-117. [CrossRef] [PubMed]

156. Grand View Research. Deep Learning Market Size E Growth, Industry Research Report 2025; Grand View Research: Pune, India, 2017.

157. Department of Infrastructure and Regional Development. Traffic and Congestion Cost Trends for Australian Capital Cities; No. Information Sheet 74; Department of Infrastructure and Regional Development: Canberra, Australia, 2015; pp. 1-39.

(C) 2019 by the authors. Licensee MDPI, Basel, Switzerland. This article is an open access article distributed under the terms and conditions of the Creative Commons Attribution (CC BY) license (http:// creativecommons.org/licenses/by/4.0/). 